\title{
Morphogenic Effects of Halogenated Thymidine Analogues on Drosophila. I. Quantitative Analysis of Lesions Induced by 5-Bromodeoxyuridine and 5-Fluorouracil
}

\author{
T. M. Rizki, ${ }^{1}$ R. M. Rizki, ${ }^{1}$ and H. A. Douthit ${ }^{2}$
}

Received 26 March 1971-Final 22 July 1971

When 5-fluorouracil (FU) is offered simultaneously with 5-bromodeoxyuridine (BUdR) to Drosophila larvae, a variety of bristle modifications and hyperplastic growths are found on the wings of the adult flies. Administration of $F U$ alone will not stimulate growth in Drosophila, while high concentrations of BUdR offered alone will induce a lower frequency of growth modifications than induced by BUdR plus $F U$. Comparison of the morphological response induced by sequential treatment with the two analogues and that by simultaneous treatment with the analogues at the same concentrations indicates that maximum response is achieved by the presence of both analogues at the same time. These observations suggest that BUdR may be the primary agent in upsetting morphogenesis in Drosophila, while FU plays a subsidiary role leading to intensification of the morphogenic effects when it is present during the treatment period. The incorporation of BUdR- $H^{3}$ and $F U-H^{3}$ in Drosophila tissues was demonstrated by autoradiography. BUdR $-H^{3}$ was incorporated in nuclei of both larval and imaginal disc cells, and the isotopic label was removable by deoxyribonuclease. Following dietary administration of $F U-H^{3}$, tritium was found in $R N A$, primarily in cytoplasmic regions. Since BUdR is a known mutagen, consideration was given to the hypothesis that the altered growth patterns in Drosophila wings are the result of somatic cell mutational events induced by BUdR. Validity of the argument that recessive mutations on the $X$ chromosomes can be readily expressed in the somatic cells of the male with one $X$ chromosome as opposed to the female with two $X$ chromosomes was tested by comparing the frequency of the induced somatic cell lesions in male and female zygotes. The males showed a higher frequency of induced supernumeraries, while the incidence of bristle effects and total wings affected was the same in both sexes.

\section{INTRODUCTION}

It was previously reported that oral administration of a mixture of 5-bromodeoxy-

\footnotetext{
This research was supported by grants to T. M. R. from the National Science Foundation (GB11745 ) and by an Institutional Research Grant (No. IN-40H) to the University of Michigan from the American Cancer Society.

${ }^{1}$ Department of Zoology, University of Michigan, Ann Arbor, Michigan.

${ }^{2}$ Department of Botany, University of Michigan, Ann Arbor, Michigan.
} 
uridine (BUdR) and 5-fluorouracil (FU) to Drosophila larvae altered development of adult structures (Rizki and Rizki, 1969). Prior to attempting a causal analysis of molecular upset and developmental modifications in Drosophila, precise information on the conditions inducing the various morphological deviations was necessary, and the present report details these studies. In addition, the cellular distribution pattern of analogue incorporation into DNA and RNA of treated Drosophila zygotes was examined by autoradiographic techniques.

\section{MATERIAL AND METHODS}

Procedures for raising the Drosophila melanogaster (Ore-R) larvae and administration of the analogues were the same as those used previously (Rizki and Rizki, 1969). Larvae were maintained on Cream of Wheat Drosophila medium until they were 50 $52 \mathrm{hr}$ of age. They were then washed with $1 \% \mathrm{NaClO}_{4}$, rinsed with distilled water, and transferred to plastic beakers containing Whatman paper pulp moistened with the analogue solutions. The concentration of analogue solutions (in distilled water) used and duration of treatment are presented for each experiment in the following section. At the completion of analogue treatment, the larvae were rinsed briefly with distilled water and returned to vials containing Cream of Wheat medium seeded with Fleischmann's yeast. The adults were examined within 2 days after hatching. Wings were removed and mounted on slides for microscopic examination as described previously (Rizki and Rizki, 1969).

BUdR-H ${ }^{3}$ (New England Nuclear Corp.; specific activity $8.95 \mathrm{c} / \mathrm{mmole}$ ) and FU- $\mathrm{H}^{3}$ (New England Nuclear Corp.; specific activity $5 \mathrm{c} / \mathrm{mmole}$ ) were used at $0.025 \mathrm{mc} / \mathrm{ml}$. Larvae were placed in containers with an aqueous solution of the radioisotopes and paper pulp when they were $50 \mathrm{hr}$ of age. After a period of $5 \mathrm{hr}$ on the moist paper pulp, the larvae were rinsed and returned to regular food dishes. Specimens were removed and fixed in Carnoy solution at 68, 93, 96, and $120 \mathrm{hr}$. They were embedded in paraffin and sectioned at $5 \mu$. Slides intended for digestion studies with deoxyribonuclease and ribonuclease were mounted in paired series so that alternate sections would be available to serve as control material for the sections which would be exposed to enzyme solutions. Sections were placed in $0.1 \mathrm{mg} / \mathrm{ml}$ deoxyribonuclease (Worthington Biochemical Corp.) in $0.01 \mathrm{M}$ phosphate buffer containing $0.003 \mathrm{M}$ $\mathrm{MgSO}_{4}$ at $p \mathrm{H}$ 6.4. Control sections remained in the phosphate- $\mathrm{MgSO}_{4}$ solution for the same period of $1 \mathrm{hr}$ at $37 \mathrm{C}$. Several slides from each group were stained with Feulgen to verify the effectiveness of the deoxyribonuclease treatment. Ribonuclease (Worthington Biochemical Corp.) was used in distilled water at a concentration of $0.1 \mathrm{mg} / \mathrm{ml}$ at $p \mathrm{H} 6.5$ for $1 \mathrm{hr}$ at $37 \mathrm{C}$. Control slides were placed in distilled water for $1 \mathrm{hr}$. Decrease in basophilia following ribonuclease digestion was noted by staining treated and control slides with $0.025 \%$ Azure $\mathrm{B}$ at $p \mathrm{H} 4$.

Slides were coated with Kodak NTB3 nuclear track emulsion at $43 \mathrm{C}$ and placed in an atmosphere of circulating warm air for $1 \mathrm{hr}$ until they were thoroughly dry. They were then transferred to a desiccator and stored in a refrigerator for 9 days before development of the latent image.

\section{RESULTS}

\section{Autoradiography}

Autoradiographs prepared from larvae which had been given a 5-hr pulse of BUdR- 
Fig. 1. Autoradiographs of Drosophila larvae fed BUdR-H ${ }^{3}$ plus FU. A: Autoradiograph of a segment of ovarian tissue and a fat cell. This section was stained with Feulgen to contrast nuclei and cytoplasm underlying the autoradiographic emulsion. Radioactive foci appear over the nuclei of some of the ovarian cells (oc) and are concentrated over the single fat cell nucleus (fn) in the lower right. The ovarian cell nuclei are $4 \mu$ in diameter, whereas the fat cell nuclei containing polytene chromosomes average $15 \mu$. The extent of the cytoplasm of the fat cell occupying the lower diagonal half of the photographic field is indicated $(\mathrm{fc})$. $\times 3000$; reduced $50 \%$ for reproduction. B: A larval gut "cuprophilic" cell photographed under phase contrast. The lumen of the gut contains partially digested yeast cells $(y)$ since this larva was fixed immediately after removal from the food surface. $\times 1000$; reduced $50 \%$ for reproduction. $\mathrm{C}$ : The cell appearing in $\mathrm{B}$ showing radioactive foci over the nucleus. Radioactivity does not appear in the gut contents. $\times 1000$; reduced $50 \%$ for reproduction.

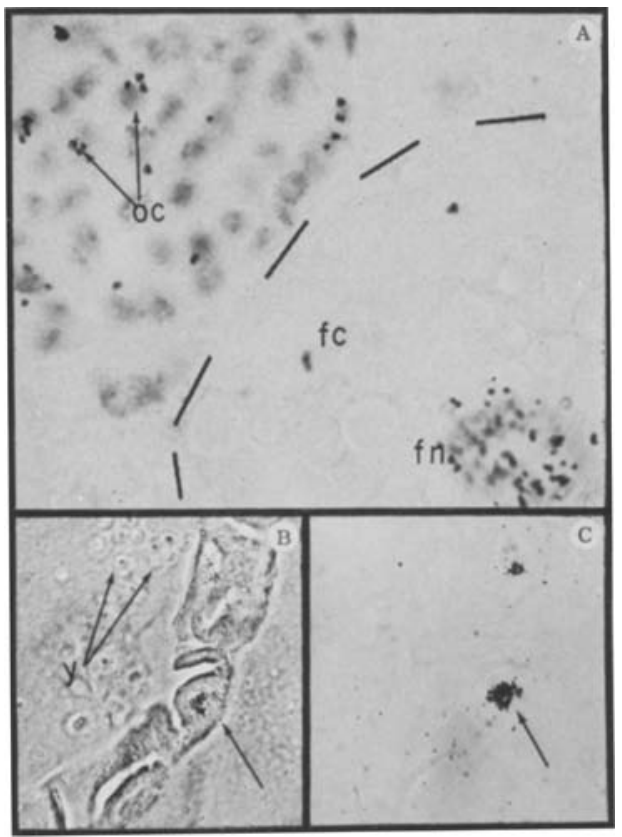

$\mathrm{H}^{3}$ showed the tritium label in nuclei of both larval and imaginal tissues. Asynchrony in incorporation of the label among the cells was particularly striking in larval tissues with polytene chromosomes such as the fat cells and gut cells, and nuclei lacking label were interspersed among nuclei which had incorporated BUdR heavily (Fig. 1). A similar dispersion of label among the cells of the imaginal discs was noted (Fig. 2). This pattern of label distribution indicating incorporation into a limited fraction of the cell population was observed in the specimens which were removed from the BUdR$\mathrm{H}^{3}$ and allowed to continue development on normal medium until they completed the third larval instar and entered pupation. BUdR which is incorporated into DNA early in the third larval instar is thus retained, and cells containing the analogue are present at the time of differentiation of the imaginal tissues in the pupal stage. No distinction between label distribution among various cell types was noted in specimens receiving $\mathrm{FU}$ with $\mathrm{BUdR}-\mathrm{H}^{3}$ and those fed only $\mathrm{BUdR}-\mathrm{H}^{3}$. The tritium label in both instances was removed by deoxyribonuclease digestion.

In the autoradiographs of larvae fed $\mathrm{FU}-\mathrm{H}^{3}$ at $50-55 \mathrm{hr}$ of age and then fixed 12 hr later, cytoplasm showed extensive label which was removable by ribonuclease. This cytoplasmic label was still present in larvae at the time of pupation. Control groups of larvae for this experiment were given BUdR with $\mathrm{FU}-\mathrm{H}^{3}$, and the presence of BUdR during the pulse treatment with $\mathrm{FU}-\mathrm{H}^{3}$ did not produce any noticeable differences in incorporation pattern.

\section{Morphogenic Characteristics of Induced Lesions}

The effects of BUdR plus FU treatment may be manifested in exoskeletal structures throughout the adult flies, and quantitative comparison of specimens is thus extremely difficult. This difficulty was circumvented in our previous study (Rizki and Rizki, 


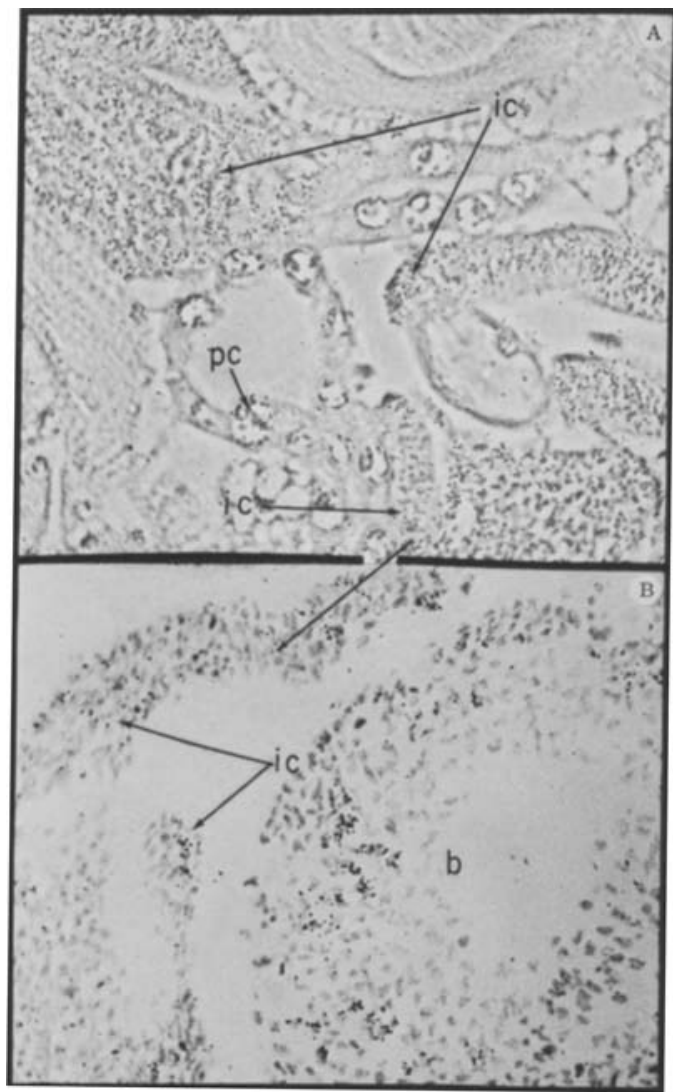

Fig. 2. Autoradiographs of Drosophila larvae fed BUdR $-\mathrm{H}^{3}$ plus FU. A: Autoradiograph of a section of a larva fed BUdR- $\mathrm{H}^{3}$ plus $\mathrm{FU}$ at $50-55 \mathrm{hr}$ of age and fixed at $68 \mathrm{hr}$ of age. Note the incorporation of label in the imaginal discs of the cephalic complex (ic) and various other cells with polytene chromosomes (pc). Phase contrast. $\times 750$; reduced $50 \%$ for reproduction. B: Autoradiograph of a larva from the same pulse-labeled group as that in A. This specimen was fixed at the time of puparium formation, or $40 \mathrm{hr}$ after the pulse had been given. Extensive growth of the imaginal discs has taken place by cell multiplication. Compare the density of the radioactive foci in the eye antenna disc located in the lower right of $\mathrm{A}$ with an area of the eye antenna disc designated in B. The brain is indicated (b). $\times 1200$; reduced $50 \%$ for reproduction. 
1969) by scoring lesions in isolated wings, and all observations in the present study have been limited to this tissue. The types of growth effects on the wings following BUdR plus FU treatment have been grouped into three categories: modification of a single bristle, clusters of abnormal bristles, and supernumerary growth. These developmental modifications are the same as those reported previously (Rizki and Rizki, 1969), but stress should be placed on the fact that they represent increased growth and not inhibition in the development of a structure. The photographs in Figs. 3 and 4 illustrate the types of growth lesions which are considered as neoplastic growths induced by BUdR plus FU treatment. Features such as facet derangement and notched eyes, interruptions in wing veins, and incomplete or cut wing effects can be readily induced in Drosophila by a variety of environmental agents, including FU, and such modifications are generally due to the presence of isoalleles, subthreshold genes, or phenocopy effects; such inhibitory alterations are not the distinguishing characteristic of BUdR plus FU treatment. This particular combination induces growth and was therefore reported as an unusual phenomenon in Drosophila (Rizki and Rizki, 1969).

Each of the modified growths may also be classified with reference to the differentiative events described by Hadorn (1965) in their extensive studies on imaginal disc tissue of Drosophila. "Autotypic" differentiation refers to the expression of the embryonic capacities of a group of cells in agreement with the fate expected of those cells in normal development; for example, a wing disc isolated from a larva and implanted in a host larva will differentiate during pupation as wing. Following a lengthy series of consecutive implantation tests, differentiation of implanted tissue may occasionally proceed along a new path, and this alteration is designated as "allotypic" differentiation. The appearance of allotypic tissues is considered the result of a change in the original inherited state of cell determination, or a transdetermination event. Some excellent examples of both autotypic and allotypic differentiation of supernumerary tissue growth have been found in the BUdR plus FU treated specimens of Drosophila.

The specimens shown in Fig. 3A-D are examples of autotypic differentiation induced by BUdR plus FU' treatment. Supernumerary growths arising from wing tissue have undergone differentiation during metamorphosis and clearly display characteristic features of wing tissue. Examples of allotypic differentiation are shown by the two specimens in Fig. 3E, F. In these flies, supernumerary winglike structures have appeared from the dorsolateral margin of the scutellum of the mesothorax, and both of these specimens have fully developed wings in the normal ventrolateral position of the middle thorax. Thoracic tissue which is not ordinarily associated with wing development has apparently given rise to wing tissue. Additional examples of allotypic differentiation are presented in Fig. 4A-E, but in these instances the tissue growths have undergone differentiation during metamorphosis to produce structures which are foreign with reference to normal features occurring in the fly. The variety of developmental potential that may be released is indicated by the sequence of photographs in Fig. 4A-C. Neoplastic growths are found in the same general area of the wing in each of the three different specimens, and yet the quality of differentiation of the tissues is not systematic.

This phenomenon of allotypic differentiation can extend to the level of a single bristle, in contrast to the morphogenesis of a tissue mass, and is nicely demonstrated by the appearance of a single bristle from the anterior margin of the wing which resembles a large scutellar bristle of the thorax rather than any bristle normal for wing (Fig. 4F). The cluster of bristles and the single bristle depicted in Fig.4G, $\mathrm{H}$ are 


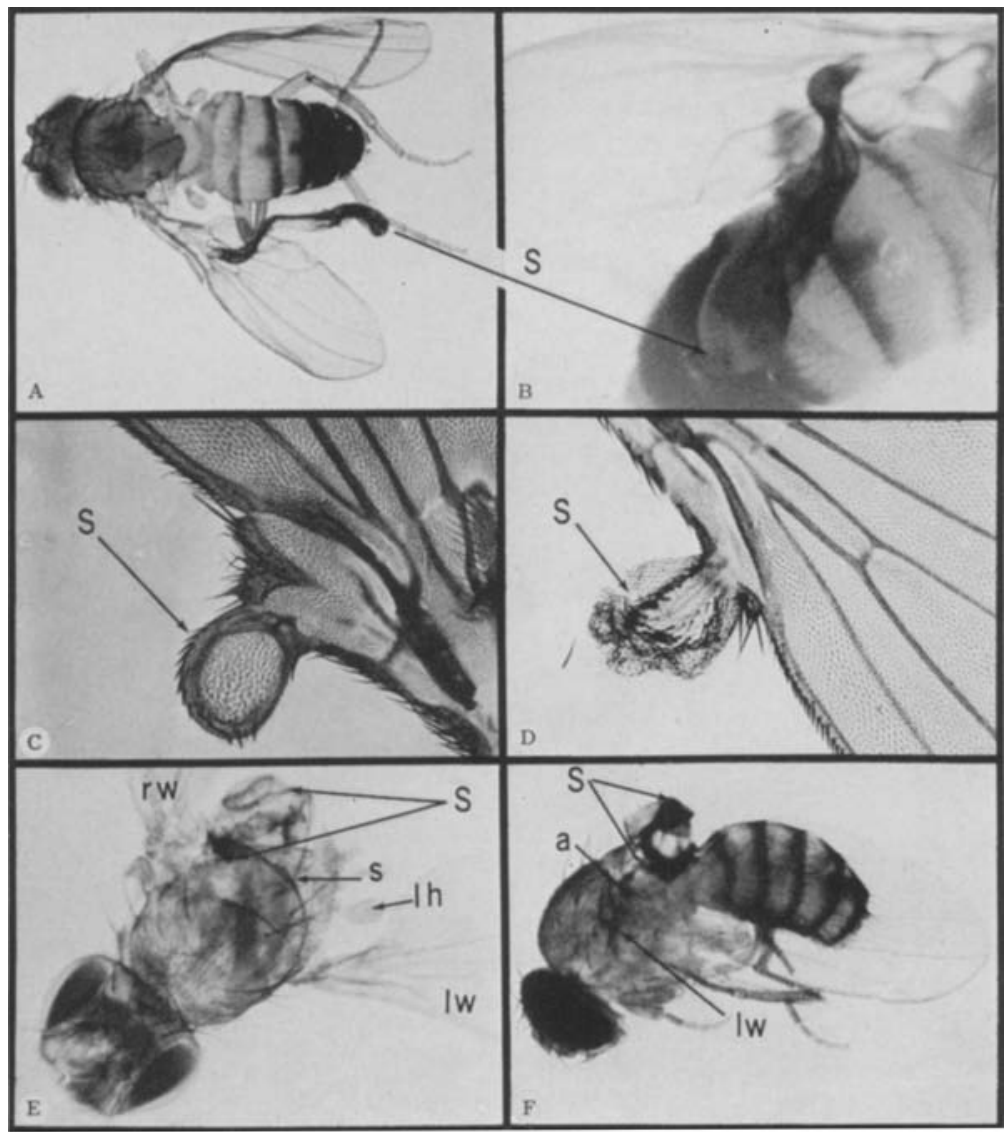

Fig. 3. Lesions induced by BUdR plus FU treatment. A: A well-differentiated autotypic structure in the form of a supernumerary (S) wing arising from the proximal region of the affected wing as compared to the unaffected right wing. The specimen is suspended in clove oil and photographed with strobic illumination. B: The same specimen as in A has been tilted to show the degree of differentiation in the supernumerary wing tissue including wing veins. $C$ and $D$ : Two examples of autotypic growths from the right wing of a fly and the left wing of another fly. The tissue development resembles wing in terms of wing hair development, texture, marginal bristles, and marginal thickening. E: Allotypic differentiation of neoplastic growth which has taken place on the right half of the thorax (scutellum) and replaced the scutellar bristles, which are labeled on the left side (s). This fly has a normal right wing (rw) and left wing (lw) and normal halteres, the left one of which is labeled (lh). The tissue growth shows structural resemblance to wing tissue. F: A supernumerary structure growing from the left part of the scutellum. The left wing has been removed to show the attachment site of the supernumerary (a), and the attachment site of the normal left wing is indicated (lw). A region of this supernumerary growth has melanized, but the tissue development clearly resembles wing. 


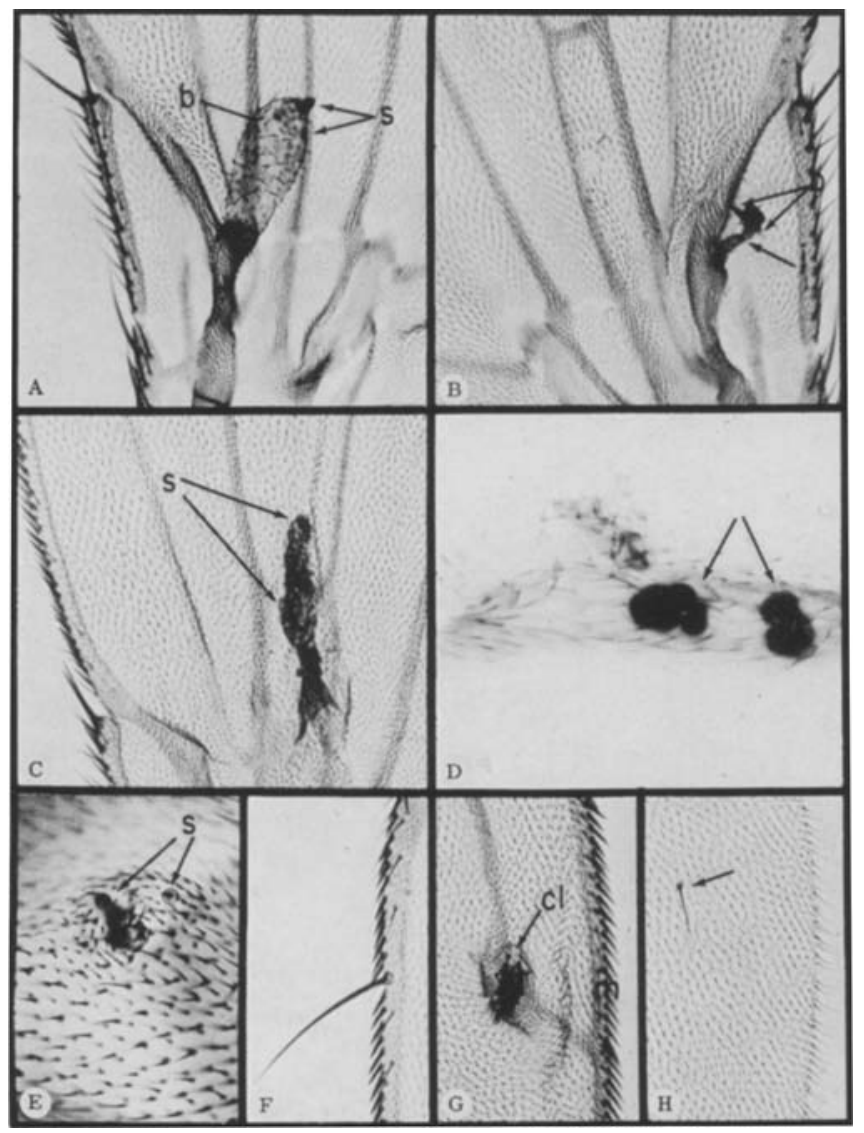

Fig. 4. Lesions induced by BUdR plus FU treatment. A: A clubshaped outgrowth on the wing. The structure shows development of sense organs (s), and one of the long bristles accompanied by a short branch (b) is the type commonly found on the leg of the fly. B: An allotypic structure on the wing showing joints (j) and a corona with a series of well-formed bristles, only two of which are in focus (b) since the others are facing upward or downward away from the focal plane. C: A mulberry-shaped outgrowth from a wing showing extensive development of sense organs (s). D: An enlarged view of five small supernumerary nodules with fully differentiated bristle structures embedded in the distal part of the wing vein. $\mathrm{E}$ : A supernumerary structure in the general area of the wing blade. In this area, two sense organs (s) have developed in relation to a tuft of heavier bristles protruding upward from the focal plane. $F$ : A single large bristle appearing on the margin of the wing. This bristle resembles the scutellar bristles on the thorax and can be compared with those in Fig. 3. G: A cluster of large bristles (cl) appearing near the anterior margin of the wing $(\mathrm{m})$. $\mathrm{H}$ : A single cell forming an allotypic structure amid the surrounding small hairs near the posterior margin of the wing. 
additional examples of allotypic differentiation; this bristle type resembles bristles which normally occur on wing tissue, but in flies which have been treated with BUdR plus FU these bristles appear in sites which should be occupied by a different type of small wing hair.

\section{Reexamination of Dose Response and Feeding Sequence}

The autoradiographic survey presented in the first section of this paper indicates that BUdR can be incorporated into Drosophila DNA whether FU is present or not. The preliminary report on morphogenic lesion induction in Drosophila implied that FU and BUdR must both be administered in order to induce developmental deviations, since no growth lesions were recorded when either analogue was offered alone (Rizki and Rizki, 1969). Dosage studies showed a correlation between BUdR concentration and morphogenic modifications, however, leading to the conclusion that BUdR may be the responsible agent in creating growth lesions while FU might serve in an auxiliary capacity. The available information thus far would indicate that the incorporation of BUdR into DNA may not be the necessary prerequisite for induction of growth lesions and that their induction depends upon events requiring the presence of both analogues. However, before excluding BUdR-incorporated DNA as instrumental in upsetting development, a reexamination of the morphogenic response to a variety of analogue doses was deemed necessary; the results of these experiments resolve to a certain extent the apparent discrepancy and support the suggestion that BUdR is the effective agent in inducing hyperplasia in Drosophila.

In the previous study, a 4-hr period of treatment with BUdR and FU was sufficient to induce developmental abnormalities, and a maximum response was reached at $0.15-0.20 \mathrm{mg} / \mathrm{ml}$ BUdR. Treatment for this length of time was not effective with a comparable dose of BUdR or FU alone. For the present studies, the duration of treatment was extended to $6 \mathrm{hr}$ and dosage of each analogue was raised as high as $4 \mathrm{mg} / \mathrm{ml}$. The types of lesions induced were scored in the three categories as described above, and these data are presented in Table I. Several bristle deviants were produced by treatment with $0.5 \mathrm{mg} / \mathrm{ml} \mathrm{BUdR}$ for $6 \mathrm{hr}$, and an approximately fourfold increase in this lesion type was stimulated by using $4 \mathrm{mg} / \mathrm{ml} \mathrm{BUdR}$. Higher concentration of BUdR also stimulated supernumerary development. However, no instances of super-

Table I. Morphogenic Response to 5-Fluorouracil and 5-Bromodeoxyuridine

\begin{tabular}{|c|c|c|c|c|c|c|}
\hline \multirow{2}{*}{\multicolumn{2}{|c|}{ Analogue $(\mathrm{mg} / \mathrm{ml})$}} & \multirow{3}{*}{$\begin{array}{c}\text { Wings } \\
\text { affected/total }\end{array}$} & \multicolumn{3}{|c|}{ Type of lesion } & \multirow[b]{3}{*}{ Lesion frequency } \\
\hline & & & \multirow{2}{*}{ Single } & \multirow{2}{*}{ Cluster } & \multirow{2}{*}{$\begin{array}{c}\text { Super- } \\
\text { numerary }\end{array}$} & \\
\hline BUdR & FU & & & & & \\
\hline 0.5 & - & $14 / 233$ & 13 & 0 & 1 & 0.06 \\
\hline 4.0 & - & $71 / 255$ & 54 & 1 & 49 & 0.40 \\
\hline- & 0.15 & $0 / 162$ & 0 & 0 & 0 & 0 \\
\hline- & 4.0 & $0 / 75^{b}$ & 0 & 0 & 0 & 0 \\
\hline
\end{tabular}

${ }^{a}$ A wing may contain more than one lesion; lesion frequency represents the total lesion number per total wings counted.

${ }^{b}$ In addition to 75 adults there were 40 dead pupae in this sample. 
numeraries or bristle inductions were observed in the wings from groups of flies treated with FU under these conditions even when concentration was raised to $4 \mathrm{mg} / \mathrm{ml}$. High concentrations of FU or extended intervals of treatment with this analogue cause lethality in Drosophila (Rizki, 1968).

Since combined treatment with both analogues is far more effective than even high concentration of BUdR given alone, the next question to examine was whether both analogues must be administered during the same feeding period or whether the morphogenic effects could be induced by treating larvae with one analogue at one time during development and then with the other analogue at some other time in development. Treatment at $52-56 \mathrm{hr}$ of age with $\mathrm{FU}(0.2 \mathrm{mg} / \mathrm{ml})$ was followed by treatment with BUdR $(0.5 \mathrm{mg} / \mathrm{ml})$ at $58-62 \mathrm{hr}$ of age; the reverse sequence of analogue administration was also tried. Two control groups of larvae were used for this experiment: one was exposed to BUdR plus FU during the first interval of treatment and the other was exposed at 58-62 hr. All groups were maintained under normal feeding conditions at 56-58 hr. The larvae given simultaneous treatment were placed on water for the duration of the alternate treatment period; in this way, the four groups were handled in the same manner regarding the washing and transfer procedures.

The data presented in Table II compare simultaneous treatment and sequential treatment with the two analogues. Sets $\mathrm{A}$ and $\mathrm{B}$ are independent experiments. In these experiments, the 2-hr interval of feeding between the periods of analogue treatment was included to allow removal and/or dilution of the first analogue from the gut and cell pools. No assessment of this assumption can be made, and the abnormalities obtained in groups A3, A4, and B2 may be the result of a brief period when both FU and BUdR were available in cell pools or in the lumen of the gut. On the other hand, these abnormalities may be the result of exposure to BUdR since the fraction response in these groups agrees with the response obtained with $0.5 \mathrm{mg} / \mathrm{ml} \mathrm{BUdR}$ (see Table I). The data on the abnormalities induced in the wings are presented as single bristle effects, clusters of similar deviant bristles, and supernumerary growths. The latter two categories of abnormal sites are, of course, composed of more than one cell, but each of these has again been scored as a single event. Since an individual wing may develop more than one lesion, the data are presented in terms of the total number of lesions induced for the total wing sample as well as the number of affected wings. Comparison of the fraction response for sequential and simultaneous treatment clearly demonstrates superior effectiveness of the latter.

\section{Somatic Cell Mutation}

Incorporation of BUdR into DNA leads to the production of mutations in microbial cells (Rudner, 1961; Strelzoff, 1961). Autoradiography has confirmed the incorporation of BUdR into the DNA of the imaginal disc cells of Drosophila when it is administered according to the same procedure which effectively induces morphological modifications in development. It seems reasonable, therefore, to consider the possibility that some of the morphogenic alterations in the adult wing represent the expression of mutational events due to the incorporation of BUdR into the wing cells during their development. A direct approach to testing this hypothesis is difficult with somatic cells in situ; however, a number of alternate analyses can be used on the lesion frequency data to provide some clues as to the validity of this hypothesis. One of these is a comparison of the distribution of lesions in the two sexes, since the male Droso- 


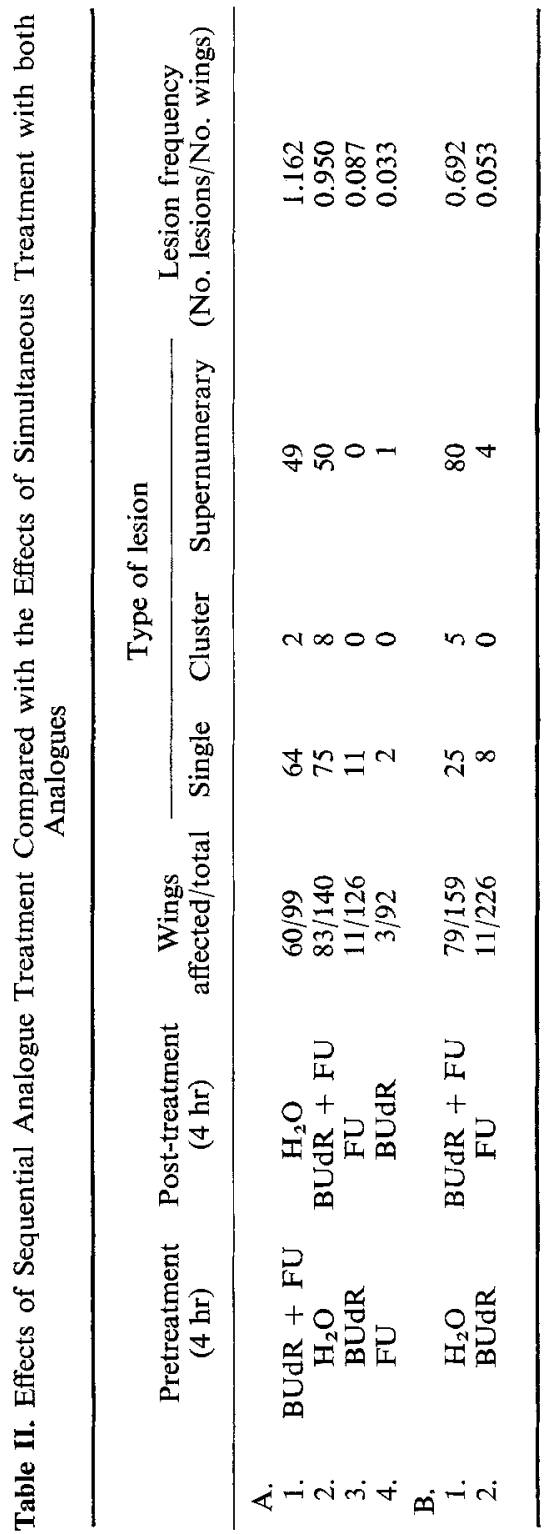


phila is heterogametic and has only one $\mathrm{X}$ chromosome. If the morphogenic effects induced by BUdR plus FU treatment are the results of genic changes, a difference in expression might be expected between cells with a single $\mathrm{X}$ chromosome and cells carrying two such chromosomes. The remainder of the chromosomal complement is the same, and differential response would not be expected between the sexes for events associated with autosomal genes. Dominant-type effects would express themselves in somatic cells of either sex whether they are autosomal or sex-linked, but the expression of recessive mutations would be limited to those on the $\mathrm{X}$ chromosome of the male somatic cells. Therefore, the frequency of mutations in the cells of male zygotes would include both dominant and recessive events, and one would predict an overall increase in the cells of the male. Such an increase in mutational events would, of course, only be detected by our system of observation if affected cells are viable and display an altered morphology either individually or in concert with other cells in the developing wing tissue. If incorporation of BUdR into specific sites of the DNA leads to cell death, a lower frequency of morphogenic lesions might be detected in the cells of the male. On the other hand, embryonic regulation may tend to cover any discrepancy between the sexes depending on the extent to which a malfunction may be replaced by surrounding cells. Such an analysis is thus inherently complicated, but can be used to provide some information about lesion induction.

Larvae were given BUdR plus FU, and lesions on the wings of adult males and females were scored separately. Approximately $50 \%$ of the wings of both sexes were affected (Table III), so no differences in overall physiological responses between males and females are detectable. A difference between the sexes becomes apparent, however, when the frequency of lesion induction is compared: 484 lesions were found in a total of 522 male wings, or a frequency of 0.927 , while 347 female wings contained only 248 lesions, or a lesion frequency of 0.715 . The increased frequency of lesions in male zygotes lies in the class of supernumerary growths, while the bristle-type effects, both single and cluster, occur with similar frequencies in both sexes.

Consideration should be directed to the cell types involved in the development of the two classes of lesions which are classified on the wing. The wing was selected for study since it represents a double-layered structure with a precise pattern of hair and bristle formation. In scoring deviation from this pattern, we can detect a change in morphology in a specific wing bristle or we can detect the formation of a bristle in a locality normally occupied by a small wing hair. Either event is a change in a hypo-

Table III. Effect of BUdR plus FU on the Wings of Females and Males ${ }^{a}$

\begin{tabular}{|c|c|c|c|c|c|c|}
\hline \multirow{2}{*}{ Sex } & \multicolumn{4}{|c|}{ Frequency of lesions ${ }^{b}$} & \multicolumn{2}{|r|}{ Wings } \\
\hline & Single & Cluster & Supernumerary & Total & Affected $^{b}$ & Total examined \\
\hline Female & $\begin{array}{l}0.337 \\
(117)\end{array}$ & $\begin{array}{c}0.060 \\
(21)\end{array}$ & $\begin{array}{l}0.317 \\
(110)\end{array}$ & $\begin{array}{c}0.715 \pm 0.029 \\
(248)\end{array}$ & $\begin{array}{l}0.50 \\
(173)\end{array}$ & 347 \\
\hline Male & $\begin{array}{l}0.333 \\
(174)\end{array}$ & $\begin{array}{c}0.075 \\
(39)\end{array}$ & $\begin{array}{l}0.519 \\
(271)\end{array}$ & $\begin{array}{c}0.927 \pm 0.012 \\
(484)\end{array}$ & $\begin{array}{c}0.52 \\
(269)\end{array}$ & 522 \\
\hline
\end{tabular}

${ }^{a}$ Comparison of the distribution of lesion types among females $(117,21,110)$ and males $(174$, 39,271 ) shows a value of $\chi^{2}{ }_{2 \mathrm{~d} f}=10.15$ with $P=0.007$.

${ }^{b}$ The numbers of lesions and affected wings are given in parentheses. Frequency of lesions and affected wings are based on total number of wings examined. 
dermal cell, presumably prior to the final differential mitoses which form the four elements comprising each wing bristle. The formation of a deviant cluster of bristles possessing the same morphology would result from changes in hypodermal cells, and it seems reasonable to consider that these may represent a clone if the BUdR-induced event was transmitted to a number of daughter cells prior to the final definitive mitoses. On the other hand, some supernumerary structures are composed of a variety of bristles, nervous elements, and hair cells, and many of them clearly display morphogenic regulation by the formation of marginal structures at the distal end. In addition, disruption of the mesenchymal elements can often be observed underlying these areas of hyperplasia. It seems likely that supernumerary induction may be related to phenomena at the intercellular level. A developmental sequence dependent on field coordination involving both mesenchymal and hypodermal cells would be disrupted by unregulated processes within any of the elements of the coordinated set of cells.

\section{DISCUSSION}

A variety of chemical as well as physical agents can modify embryonic development to produce abnormal zygotes. Generally, these alterations are the result of differential inhibition of growth of specific regions of the embryo. The stimulation of growth, as well as allotypic differentiation of regions of excessive growth in Drosophila following BUdR plus FU treatment, thus presents unusual and interesting features. These growth effects are associated with low doses and brief periods of analogue administration. Mortality of zygotes and destruction of tissues are encountered at higher doses or with lengthy intervals of treatment. We have therefore attempted to establish precise conditions which induce growth deviations so that an eventual correlation of factors at the molecular, cellular, and organismic levels may be possible.

Our analysis thus far indicates that BUdR is the primary agent in initiating hyperplasia in Drosophila since it induces morphogenic events when applied alone while FU is ineffective under the same conditions of application. The morphogenic effect is increased manyfold when FU is offered simultaneously with BUdR. The magnitude of this increase, which has been illustrated by comparing the number of lesions induced, is an underestimate of the morphogenic response, since many of the supernumerary structure induced by combined treatment are larger in size than those found in BUdR-treated specimens. Sequential treatment with the two analogues is not equivalent to simultaneous treatment, and both analogues must be present at the same time to induce maximum response. These observations would be consistent with the hypothesis that the incorporation of BUdR into Drosophila DNA creates the morphogenic disturbances and the degree of morphogenic response is correlated with the level of analogue incorporated. An increase in BUdR incorporation into DNA achieved either by raising the concentration of analogue administered or by blocking the endogenous thymidine supply for DNA synthesis would lead to increased morphogenic response. FU is converted to FUdR, and the latter has been employed extensively to block thymidylate synthesis in a variety of biological systems, thereby stimulating utilization of an exogenous source of thymidine or a thymidine analogue for DNA synthesis (Lorkiewicz and Szybalski, 1960; Simon, 1963; Haut and Taylor, 1967). It is reasonable to suspect that if FU is converted to FUdR in Drosophila, it may inhibit thymidylate synthetase and lead to increased incorporation of BUdR into Drosophila DNA. The autoradiographic studies show that there is no difference as to the type of cells incorporating BUdR into their DNA whether FU is present or not, 
so a differential distribution of analogue among cell types can be ruled out as an explanation for the difference in morphogenic response in the presence or absence of FU. Autoradiographic examination as used in the present study, however, does not exclude the possibility of quantitative differences in incorporation among the cells of a specific tissue. Studies on isolated Drosophila DNA have indicated that differences in BUdR incorporation into DNA may occur in the presence of FU (Rizki et al., submitted for publication).

Differentiation of avian cells in culture is inhibited by the presence of BUdR in the medium (Bischoff and Holtzer, 1970; Coleman et al., 1969; Lasher and Cahn, 1969). This inhibition is considered reversible since treated cells will resume their typical mode of differentiation when they are removed from BUdR. It would appear then that BUdR can affect differentiative processes whether these occur in cells in culture or in the intact organism. The latter, of course, includes increased orders of complexity involving cell interactions and cell movements coupled with the autonomous expressions of cell-specific phenotype. Perhaps some of these features may account for the differences observed between the in vitro systems and the in situ imaginal tissues of Drosophila.

The autoradiographic studies on the imaginal discs of Drosophila indicate that cells containing BUdR- $\mathrm{H}^{3}$ are retained during the remainder of larval life and are still observable in the pupal period when wing morphogenesis is occurring. Destruction of cells which have incorporated BUdR accompanied by loss of developmental homeostasis does not appear to be the significant factor in modifying differentiation in the wing. This contention is further strengthened by analogue induction of single bristle deviations, for these represent the product of a single cell in the wing. Furthermore, the time of analogue treatment is ontogenically remote from the time of differentiation and can represent a separation as great as $80 \%$ of the developmental period (Rizki and Rizki, 1969; and manuscript in preparation). Any deleterious metabolic effects evoked during the treatment period by the analogues might conceivably be overcome by homeostatic mechanisms which bring about regulation during differentiation and morphogenesis.

There is evidence that replication of DNA containing BUdR may be modified and cell division may be inhibited (Abe and Tomizawa, 1967; Haut and Taylor, 1967; Littlefield and Gould, 1960; Simon, 1963; Yoshikawa, 1970). Consideration must be directed to the asynchronous pattern of cell division in the developing imaginal disc tissues and the fact that during the pulse treatment of Drosophila larvae with BUdR, incorporation of the analogue would be limited to a fraction of the cells in an imaginal disc. A deficiency or alteration in function of one cell type may influence the differentiative behavior of other cells whose morphogenesis is correlated with the affected cells. We previously reported the observation that mesenchymal cells in an arrested state of development are often found underlying the BUdR-induced lesions. Further study of these patterns of cell interaction in normal development of the wing of Drosophila is required before we can assess this observation with relation to lesion induction.

Examination of autoradiographs of larvae fed BUdR- $\mathrm{H}^{3}$ indicates that numerous cells in the developing wing disc contain the tritium label but that an adult wing formed from such imaginal tissue may possess as little as one bristle cell deviation, while some larvae which have incorporated the analogues do not show any apparent phenotypic response as adults. Incidence of lesions in this sense is random among the individuals treated and among the cells of the affected wings. The randomness of the events as well as the low frequency of each type of lesion induced lends itself to the 
interpretation that BUdR incorporated into DNA may be mutagenic to some of the somatic cells of Drosophila. Kaufmann and Gay (1970) have demonstrated the mutagenicity of BUdR in the germ line cells of Drosophila, and there is no a priori reason to exclude mutagenicity as a property of somatic cells

According to the chemical mutagen theory of Freese (1959), BUdR-containing DNA can form mutagenic heteroduplexes and occasionally give rise to AT $\rightarrow$ GC transitions which will become permanent features of the genetic information of the cell. On the other hand, Shapiro and Chargaff (1960) have shown that in certain strains of bacteria BUdR causes a high degree of base distortion in DNA; hence BUdR incorporation may not be strictly in accordance with the concept that it serves as a thymidine analogue. Nevertheless, the overall effect of BUdR incorporation into DNA is a change in the triplet code. Even if an alteration in the genetic information does occur, its presence may not be meaningful at the translational level if the base change does not result in a functional change in protein (Alff-Steinberger, 1969). The probability of detecting a mutation at the level of cell phenotype due to a transition mediated by BUdR thus becomes very small.

If BUdR is incorporated into some critical sites of DNA thereby causing loss of genetic regulation of the cell, then that cell may show an altered phenotype. Some of the single cell modifications observed in BUdR-treated wings may be due to the presence of BUdR in the DNA of the hypodermal or mesenchymal cells of the wing. According to the semiconservative model of DNA replication, this informational change will be restricted to the progeny cell receiving the BUdR-incorporated DNA site. On the other hand, if BUdR-incorporated DNA acts as a mutagenic heteroduplex during replication, then cloning of mutated cells can occur from a single mutational event. The clusters of large bristles induced by BUdR treatment may represent clones of mutated cells. The probability of aggregation of individually affected cells appears less likely since clones of similar bristles as numerous as 13 have been observed. Unfortunately, bristle clusters represent the least frequent type of lesion induced by BUdR, and an extensive study of their size and frequency of induction with relation to age of treatment is needed for an analysis of cell lineage. The permanency of propagation of a specific phenotypic modification, i.e., mutant type, is a crucial test for establishment of a mutagenic event, and this stipulation is difficult to assess in a system of somatic cells in situ. The serial implantation techniques developed by Bodenstein (1943) and Hadorn (1965) may be useful in supplying an indirect test for mutagenicity of BUdR in Drosophila imaginal disc cells.

\section{REFERENCES}

Abe, M., and Tomizawa, J. (1967). Replication of the Escherichia coli K12 chromosome. Proc. Natl. Acad. Sci, 58:1911.

Alff-Steinberger, C. (1969). The genetic code and error transmission. Proc. Natl. Acad. Sci. 64:584.

Bischoff, R., and Holtzer, H. (1970). Inhibition of myoblast fusion after one round of DNA synthesis in 5-bromodeoxyuridine. J. Cell Biol. 44:134.

Bodenstein, D. (1943). Hormones and tissue competence in the development of Drosophilia. Biol. Bull. 84:34.

Coleman, J. R., Coleman, A. W., and Hartline, E. J. H. (1969). A clonal study of the reversible inhibition of muscle differentiation by the halogenated thymidine analog 5-bromodeoxyuridine. Develop. Biol. 19:527.

Freese, E. (1959). The specific mutagenic effect of base analogues on Phage T4. J. Mol. Biol. 1:87.

Hadorn, E. (1965). Problems of determination and transdetermination. In Genetic Control of Differentiation. Brookhaven Symp. Biol. 18:148. 
Haut, W. F., and Taylor, J. H. (1967). Studies on bromouracil deoxyriboside substitution in DNA of bean roots (Vicia faba). J. Mol. Biol. 26:389.

Kaufmann, B. P., and Gay, H. (1970). Induction by 5-bromodeoxyuridine of sex-linked lethal mutations in spermatogenous cells of Drosophila melanogaster. Mutation Res. 10:591.

Lasher, R., and Cahn, R. D. (1969). The effects of 5-bromodeoxyuridine on the differentiation of chondrocytes in vitro. Develop. Biol. 19:415.

Littlefield, J. W., and Gould, E. A. (1960). The toxic effect of 5-bromodeoxyuridine on cultured epithelial cells. J. Biol. Chem. 235:1129.

Lorkiewicz, Z., and Szybalski, W. (1960). Genetic effects of halogenated thymidine analogs incorporated during thymidylate synthetase inhibition. Biochem. Biophys. Res. Commun. 2:413.

Rizki, T. M. (1968). Site of incorporation of 5-fluorouracil in Drosophila. Genetics 60:215.

Rizki, R. M., and Rizki, T. M. (1969). Somatic cell lesions induced by the base analog 5-bromodeoxyuridine. Cancer Res. 29:201.

Rizki, R. M., Douthit, H. A., and Rizki, T. M. Morphogenic effects of halogenated thymidine analogs on Drosophila. II. Incorporation of 5-bromodeoxyuridine into DNA. Mutation Res. (in press).

Rudner, R. (1961). Mutation as an error in base pairing. I. The mutagenicity of base analogues and their incorporation into the DNA of Salmonella typhimurium. Z. Vererbungslehre 92:336.

Shapiro, H. S., and Chargaff, E. (1960). Severe distortion by 5-bromouracil of the sequence characteristics of a bacterial deoxyribonucleic acid. Nature 188:62.

Simon, E. H. (1963). Effects of 5-bromodeoxyuridine on cell division and DNA replication in HeLa cells. Exptl. Cell Res. Suppl. 9:263.

Strelzoff, E. (1961). Identification of base pairs involved in mutations induced by base analogues. Biochem. Biophys. Res. Commun. 5:384.

Yoshikawa, H. (1970). Initiation of DNA replication in Bacillus subtilis. II. Linkage between termini of parental strands and origins of daughter strands. J. Mol. Biol. 47:403. 